\title{
Communication and Information Barriers of Telemedical Applications in Emergency Situations - Emergency Doctors' Point of View
}

\author{
Martina Ziefle, Sarah Mennicken \\ Communication Science \\ Human Technology Centre \\ RWTH Aachen University Aachen, \\ Germany
}

\author{
Shirley Beul, Eva-Maria Jakobs \\ Textlinguistics and Technical \\ Communication \\ RWTH Aachen University \\ Aachen, Germany
}

\begin{abstract}
In this paper we describe information, communication, and acceptance issues in a telemedical workflow, taking a pre-hospital emergency medical service (EMS) as an example. EMS workflows are extremely time-critical, impose high responsibility on physicians, and crucially depend on close, well-trained cooperation amongst EMS personnel. Although information and communication technologies (ICT) are increasingly used to support this sensitive and life-critical process, shortcomings in the emergency workflow are still observed. This applies especially in countries in which EMS are not fully standardized (as e.g. in Germany). In this research, we empirically examined organization, communication and information gaps within EMS workflows. Together with emergency staff we schematically modeled a standard workflow circuit and visualized information, communication, and organization issues including ICT usage. Second, combining qualitative (interviews) and quantitative procedures (questionnaire), we evaluated the potential benefits and perceived drawbacks of the usage of telemedical technology in the rescue chain from the perspective of emergency physicians. Results revealed a high potential of ICT in emergency situations but its implementation has to be carefully designed. Beyond the obvious advantages, we identified critical communication and information pitfalls within this workflow. Based on these findings we derive recommendations regarding an optimization of the EMS workflow.
\end{abstract}

\section{Introduction}

Due to the demographic change, the number of ageing people who have to be provided with health care services will increase dramatically in the upcoming years [1] [2]. This especially applies to emergency cases, which increase from year to year [3]. For example in Germany, according to recent statistics, 10.2 millions rescues per year have to be accomplished, 4.7 millions of these are life-critical emergency missions [3]. In addition to raised requirements of emergency patient care, the supply chain and the availability of medical professionals continuously decline. This poses a major problem especially in rural areas, in which the density of medical care is underdeveloped [4] [5| [6].

The lack of EMS physicians leads to an alarming decrease in quality management and to deficiencies in medical care as well. Alongside, due to economic bottlenecks, the number of EMS-physician stations will be reduced, and the time of arrival of an emergency patient in the hospital is extended [3].

Thus, alternative concepts have to be developed to bridge the emerging supply gap. There is an urgent need for innovative strategies to face the current and future problems in EMS, especially in countries like Germany in which EMS workflows are still not fully standardized. Moreover, only few emergency medical interventions actually require the manual skills of an EMS-physician [3]. In most emergency situations the support of medical staff, such as paramedics, is sufficient and does not require a physician on-site.

Accordingly, as reported by [7], only a small number (14.3 \%) of rescue operations on-site factually needed an experienced EMS physician. Thus, in many emergency cases, well-qualified paramedics could care for the patient. A crossnational analysis shows that this has already been practiced in other European countries, e.g., the UK or Netherlands [3] [7].

Though EMS physicians do not necessarily have an active executive part on-site, they might still be needed in critical cases as a decision-making authority due to a greater medical expertise. Moreover, also legal regulations control the administration of medication. Paramedics need an instruction and permission of a doctor to administer medicine to a patient. However, this does not require the physical presence in the emergency scene on-site, but could be accomplished by the usage of ICT and 
telemedical care concepts in the emergency workflow [8] [9].

\section{Critical Factors within the Implementation of ICT in EMS}

One key factor for effective emergency management is designing and implementing ICT within the EMS workflow. ICT has the potential to effectively support the coordination and cooperation between staff involved in the EMS workflow.

To date, there are an upcoming number of studies reporting on the usage of ICT in the emergencyrescue-chain. In the United States, telemedical consultations between physicians in the hospitals and paramedics on-site are widely used and mostly accomplished via radio. By the use of ICT, quality of primary care and intra-hospital procedures could be optimized through a faster and more focused information transfer [10] [11] [12]. As a reaction to recent crises, as 9/11, or the hurricane Katrina in the United States, medical informatics researchers started to develop ICT as corrective measures to be used in disaster situations [13] [14].

Another innovative approach [3] in Germany aims at the broad implementation of a telematic system in EMS. The central element of the system is a competence centre with an experienced emergency medical physician, a so-called "teledoctor". Assisted by advanced mobile data transmission, all vital parameters of a patient, as well as video and pictures of the incident scene, are transferred in real-time to the competence centre. The teledoctor advises the emergency staff operating on-site (EMS-physician and/or the paramedics), providing medical knowledge as well as legal and organizational information. Also, the competence centre can support the team on-site by contacting the hospital, as well as data transfer and consultation with other institutions (e.g., family members, physicians, cardiologists, poisoning centers etc.). Thus, EMS personnel on-site can predominately focus on the main task - patients' care - without being distracted by multifaceted demands of an emergency situation. Across studies, a major requirement is high quality of ICT data reliability, security and safety, when designing innovative interactive systems for emergency response in a major incident [15]. Also, the importance of high-quality audio in the noisy environment of hospitals' emergency departments had been stressed by [16].

Nowadays, it is widely accepted that ICT plays an increasingly important role in EMS and the workflow in hospital emergency departments. But still, only few studies to date concentrate on communicative and organizational issues or coordination and cooperation of the different persons involved in the rescue chain. In a very recent study [17], the coordination between a hospital emergency department and EMS team in the United States was examined, uncovering the enormous importance of social and communication aspects in the EMS workflow. Sociotechnical aspects, as introduced by [18] in the EMS-context are especially multifaceted and highly complex, accompanied by a high time pressure and responsibility. Authors [17] claim that the usage of ICT within the EMS process must be based on a thorough understanding of the workflow, and should face the potential areas of breakdown in the coordination between emergency personnel. In addition, the human factor and the interaction of communication and interaction of humans with technology is an extremely important success factor, which must be considered in technology-supported EMS settings [19] [20] [21]. Among other factors, the technical competence of EMS staff, but also their abilities and acceptance barriers towards technology usage should be carefully studied prior to implementing a technology in such a sensitive area [22] [23] [24] [25] [26]. It should be taken into account that a successful rollout of technology into a high-risk application area crucially depends on the acceptance of technology in, i.e. the ease of using the technology and the perceived usefulness of technology for the workflow [26] [27] [28] [29] [30].

\subsection{Research Questions}

The majority of studies concerning ICT usage in EMS context so far concentrated on the usage of technology as such. Few studies focused on organizational issues and communicative needs within different emergency teams [17]. Yet, the focus of studies dealing with emergency cases is mainly patient-centered [31], while the perspective of emergency doctors has been widely neglected, even though s/he is the main actor taking full responsibility for treatment, organization of the whole operation, and team coordination [32].

Thus, in this study, we explicitly focus on the emergency doctor's perspective, and the information and communicative needs in an emergency workflow. Based on this we analyze potential barriers and breakdowns from the perspective of emergency personnel, i.e. physicians and paramedics. We explore the influence of telemedical technology introduced into an established workflow within the German EMS system and identify perceived benefits of telemedical systems exemplarily represented by the teledoctor system.

Before integrating the teledoctor into the rescue system, the traditional emergency workflow has to be analyzed. When the ambulance is called two paramedics are sent on-site in an emergency vehicle. Depending on the severity of the incident, another vehicle is sent out to bring an additional paramedic and an emergency doctor. After the paramedics' 
arrival, a teledoctor could give them advice until the emergency doctor appears or even support her/him.

First, the current concept has to be modeled out of an emergency doctor's view. Second, communicative and organizational deficiencies of the present system have to be investigated. Finally, key requirements and demands for the successful launching of a telemedical service must be identified.

\section{Organizational Workflow}

The course of events in an emergency situation usually starts with a person calling the ambulance and talking to the staff in the primary control unit. They will forward some keywords describing the incident to the medical staff. Two paramedics will be sent to the incident site driving an ambulance car. Sometimes, a second car with the emergency physician and another paramedic is sent out.

The telemedical system [3] includes a teledoctor who has access to further information as online databases, e.g., with detailed information on intoxication, or contact to the patient's general practitioner. The teledoctor can support the staff onsite in two difference scenarios:

Scenario 1 (Figure 1): the emergency physician is in contact with the teledoctor.

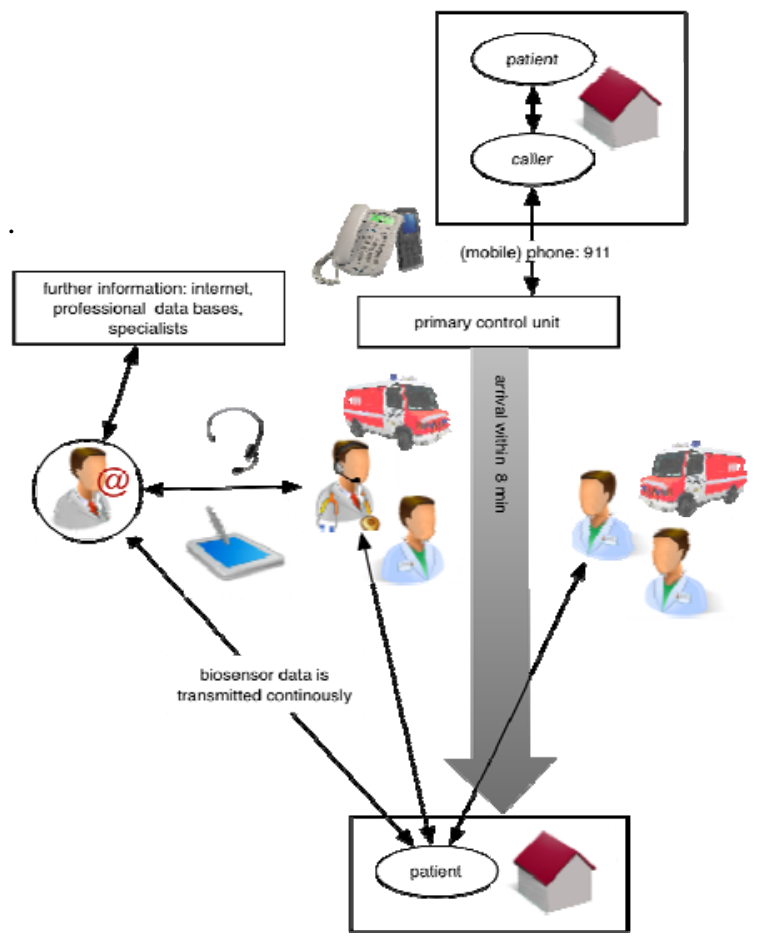

Figure 1. Emergency doctor on-site consults teledoctor

With access to an extensive range of information and her/his own experience and knowledge the teledoctor can help the emergency physician on-site.
Scenario 2 (Figure 2): one of the two paramedics onsite is in contact with the teledoctor. S/he can consult them and authorize actions that paramedics are legally just allowed to execute when being instructed by a physician.

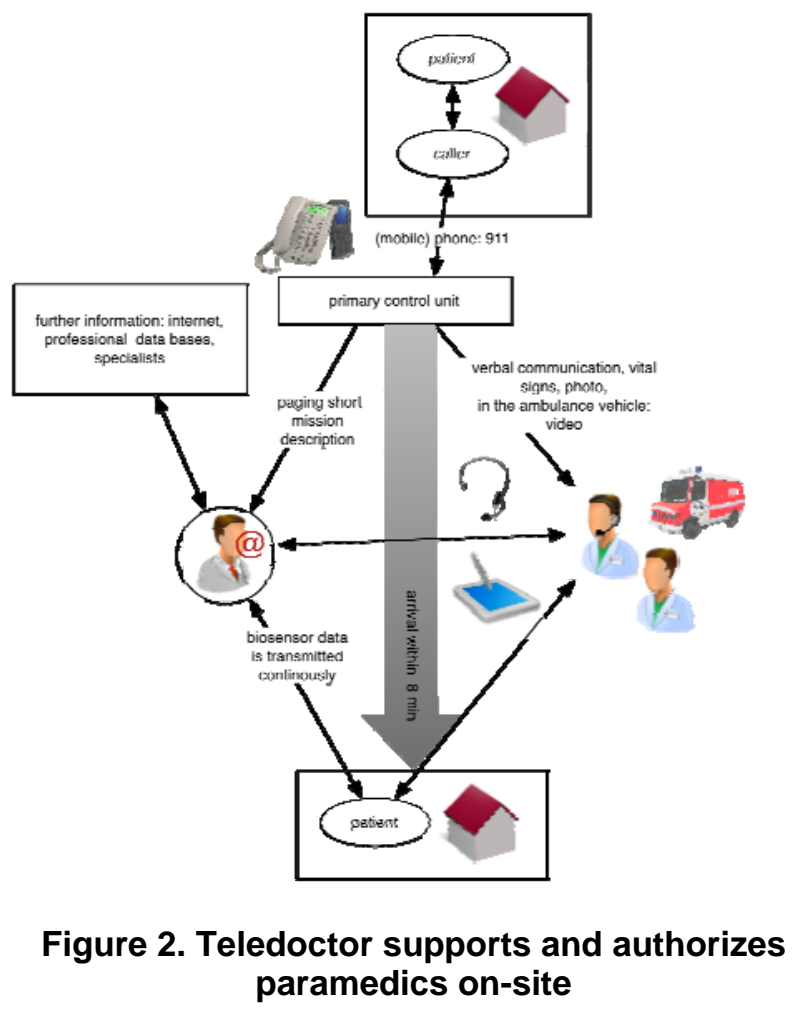

In both scenarios information on the patient's vital signs is transmitted continuously and in real-time to the teledoctor, who can talk via headset with the communication partner on-site. The staff on-site can take static images of the patient or details of the site using the camera of a tablet PC. They can enter information about the patient, the incident, and performed medical treatments into a software application. The teledoctor can see this information as well and use it to make a diagnosis.

To reveal information and communication shortcomings between the different parties involved in the "emergency call-rescue-patient care" chain, we need a modeled visualization of the multiple parties, who need to communicate for the purpose of patient care. An emergency case is a situation primarily characterized by a high risk and varying conditions: the involved actors, in particular the emergency doctor, paramedics, the patient her-/himself and her/his relatives, differ from case to case. Also, the rescue operation varies in time, location, information, organization, communication, and finally in the patient's symptoms. 


\section{Analysis of Information Flow and Usage of Technology}

In emergency situations, a lot of dynamic changes in the situation cause the need for controlled information and communication flows. Thus, a flawless flow is crucial to the efficiency of the merging actions in an emergency situation. In the following we describe how we analyzed the model described earlier and discuss our findings.

The implementation of additional technology into the emergency chain might has led to a number of benefits (i.e. efficiency increase of the communication process between doctor on-site and hospital, a more focused diagnosis, etc.), but at the same time also to a number of potential drawbacks (e.g. the problem of missing technical competence in medical doctors, additional costs which outnumber benefits). This is due to the fact that the complexity of the emergency situation relies on a number of positive and negative factors, which have to be considered at the same time. For example, the inclusion of information and communication technology might lead to benefits but also to drawbacks depending on the respective perspective: The benefit of a faster workflow by the introduction of technology (advantageous for all persons involved) might at the same time impose a higher usability demand when handling the technology (disadvantageous for emergency doctors) and lead to a higher attention load, where cognitive resources of emergency doctors are to be allocated between the treatment of the patient on-site and the handling of the technology (disadvantageous for the patient).

Therefore it is crucial to learn how experts evaluate the benefits of implementing technology into the emergency chain and the perceived drawbacks. This would allow us to determine the extent of acceptance for telemedical services as well as to identify the nature of the barriers and need of action in terms of education, information, and communication.

\section{Methodology of Empirical Approach}

In order to understand the experts' perspective on the usefulness of telemedical services during the rescue operation, we conducted an empirical study with emergency doctors with varying experience in rescue operations. A two-step procedure was pursued, combining a qualitative and a quantitative approach.

\subsection{Semi-standardized Interviews}

First, to get a deeper insight into acceptance issues and the reasoning for it, we ran semi-standardized interviews with medical professionals. After the assessment of demographic information (age, professional expertise, number of emergency rescues completed), participants detailed demands of a rescue action, different steps to be completed one after another and information and communication duties between and across the involved personnel.

Participants were requested to characterize pitfalls and shortcomings of the current situation and identify optimization potential of organization and communication of the emergency rescue operation. Furthermore, they were asked to evaluate at which points within the rescue chain additional information and communication delivered by technology could be helpful in order to enable a more efficient, less defective, and less stressful operation.

\subsection{Questionnaire}

In a next step, participants answered a questionnaire, in which they evaluated benefits and drawbacks that are connected to the usage of ICT within the rescue chain. The questions raised in the questionnaire were collected in a previous study (focus groups with emergency doctors). The questionnaire had to be answered after participants finished the interview session. The combination of the qualitative (interview) and quantitative (questionnaire) approach allowed us to understand the individual reasoning behind the evaluation out of the perspective of a group of high professionals that know the challenges of rescue operations very well and thus speak from their own experience on-site.

The completion of the questionnaire took about 10-15 minutes. In Table 1, the questions to be answered are exemplified as well as the answering modes.

\subsection{Participants}

As participants, emergency doctors ( $\mathrm{n}=11,2$ female, 9 male) volunteered to take part. They work as doctors and are on duty in several departments of hospitals (e.g. intensive care unit, operating room etc.). The age range was between $32-54$ years ( $M=$ 38.2; $\mathrm{SD}=5.9$ ). Also, the level of experience varied across participants, ranging from 2 to 25 years $(\mathrm{M}=$ 8.4 ; SD = 6.2).

All physicians take shifts in rescue services and therefore they are practicing emergency doctors. Partially, they have had experience with telemedical support $(n=4)$.

Participants were not gratified for their efforts. They showed a high willingness to participate in the study, because they reported to welcome a usability study, which focuses on the perspective of medial represented doctors and takes their perspectives seriously (especially as they have full medical responsibility on-site, experience the time pressure and have an expert view on the topic). 
Table 1. Questionnaire examples and used scale

\begin{tabular}{l|l}
\hline \multicolumn{1}{|c}{ Questions } & \multicolumn{1}{c}{ Scale } \\
\hline $\begin{array}{l}\text { Which medical operations could be supported to which degree by } \\
\text { telemedical services during a rescue operation? } \\
\text { (ECG, auscultation, documentation support, contact to hospital, therapy } \\
\text { guidelines, access to medical data libraries and compendia, accessibility of an } \\
\text { experienced colleague all day and night) }\end{array}$ & $\begin{array}{l}\text { (6-point Likert-scale) } \\
\text { from 'very high' to 'very low' }\end{array}$ \\
\hline $\begin{array}{l}\text { How big are expected gains by the usage of telemedical services in } \\
\text { emergency medicine? } \\
\text { (increased efficiency, decreased costs, better care, higher diagnostic power, } \\
\text { better document standardization, fewer false alarms, faster data transmission) }\end{array}$ & $\begin{array}{l}\text { (6-point Likert-scale) } \\
\text { from 'very high' to 'very low' }\end{array}$ \\
\hline $\begin{array}{l}\text { How big are expected drawbacks by the usage of telemedical } \\
\text { services in emergency medicine? } \\
\text { (insufficient technical competence, increase in external control, reduced } \\
\text { willingness to take on responsibility, increased time effort, increased need of } \\
\text { qualified emergency doctors) }\end{array}$ & $\begin{array}{l}\text { (6-point Likert-scale) } \\
\text { from 'very high' to 'very low' }\end{array}$ \\
\hline $\begin{array}{l}\text { Potential benefits of telemedical services outperform potential costs. } \\
\text { (6) }\end{array}$ & $\begin{array}{l}\text { from 'fully agree' to 'totally } \\
\text { (6-point Likert-scale) }\end{array}$ \\
\hline
\end{tabular}

\section{Results}

The result section first reports on insights from the semi-standardized interviews. Here, we delineate the most prominent arguments of emergency doctors and substantiate them by some original quotes. In a second step, we outline the findings of the questionnaire: first, we describe potential emergency applications, which might profit from ICT usage. Then we report emergency doctors' perceived gains and drawbacks as well as vulnerable keystones in the traditional emergency workflow that should be optimized.

\subsection{Qualitative Findings (Interviews)}

Respondents stated the following tasks as communicative and organizational tasks of an ED during a rescue operation: investigation of indispensable information, supervision of the rescue team, self-coordination, organization of single actions of the medical treatment in appropriate timeslots while following default schemes depending on the symptoms. Alongside, EDs have to take care of the patient, and her/his family members. After analyzing the information flow in the current EMS, the integration of telemedical elements in the rescue chain is discussed in consideration of acceptance and organization issues.

\subsubsection{Information Flow in the Current EMS}

After getting the alarm signal, the emergency physician receives only sparse information about the incident as such. The information is mostly limited to logistic facts, and only few details about the patient's condition are given. In special cases, additional information is provided, for e.g. the age of the patient (pediatric emergency). Participants reported that the transmitted medical details often turn out not only to be incomplete but even false. Owing to the fact that most people can only interpret symptoms based on personal assumptions and are basically in an emotional state of emergency the real symptoms differ in most cases from the announced.
„If it is correct what he [the caller] is telling on the phone, then yes [the information is helpful]. But mostly, it does not really occur what was announced in the alarm report. [...] Finally, if you open the door and the first person on-site is telling you something, then I know I get information. You can imagine the rough direction, but you can never know the situation [before your arrival]“ $\left(\mathrm{ED}_{3}\right)$.

On-site, the ED has to deduce the patient's anamnesis by interviewing him/her (or relatives in case the patient is unconscious or in a serious condition). However, the quality of this information varies: in many cases people are not able to give reliable information about the patient's medical history because of communicative problems (e.g. a non-receptive patient).

\footnotetext{
"The patient is in a state of emergency as a general rule. This means he is hard to handle. They are literally shocked [...] and answer questions not always truthfully. Not because of malevolence, because they are not getting it [the question]. I often recognize this: the first anamnesis is usually accomplished by the arriving team, the second anamnesis is done by the arriving physician, implies a plus b. The anamnesis or the first talk with the doctor in the hospital includes a, and $\mathrm{b}$ and leads to [the new anamnesis] c. So, it is
} 
becoming more and more. When patients are feeling safe, getting treated, then they remember 'oh yes, I've had a heart attack before'. But when you have asked them before, they negated it clearly“ $\left(\mathrm{ED}_{8}\right)$.

Usually, the patient's general practitioner documents medical history continuously. Emergency doctors wish quite often they could have access to these data to get a fast and precise overview. Presently, they rely mainly on data given by the patient or her/his relatives. In any case, the rescue team looks for medical documents, e.g. medication lists, letters of hospitals or surgeries etc. in the patient's immediate proximity. When an old person is in need of medical aid, the (physical) absence of a living will aggravates the situation, since emergency doctors need to respect a person's last will $\left(\mathrm{ER}_{1}\right)$. Apart from gathering information from people and collecting available documents, the rescue team investigates the emergency location (external anamnesis). If an intoxication is assumed for instance, the rescue team investigates the location for drugs, other toxic substances etc. to collect more information.

The rescue team examines the patient and measures his vital data. The ascertained patient condition as well as the collected data serves as a basis for decision-making, diagnosis, and treatment. Due to the fact that EDs are specialists from all kinds of medical branches (mainly from anesthesiology, but also surgery, internal medicine), their specialized expertise varies. An experienced ED is able to deal with most types of emergency cases. But in case their medical knowhow is not sufficient, three major compensation strategies come to the fore: (1) looking the issue up in a medical pocket book, (2) if possible, calling an expert of the required medical branch or a more experienced ED and asking for advice, (3) applying the "load-and-go"-strategy, which means the patient is transported to the hospital immediately.

After or within the emergency treatment, the ED has to check the most suitable hospital with capacity for the patient's further medical care. The ED gives the hospital relevant medical information in advance. After transporting the patient to the emergency department, the ED delivers the patient and the operation report including the collected data.

\subsubsection{Telemedical Support in the Rescue Chain}

Participants without experience with telemedical support during emergency operations revealed to be very reluctant to technology usage within the rescue chain. They reported not to be sure if technology would be safe enough and are afraid of medial problems of the teledoctor concept. "A photo would be too static to provide a reliable impression of the patient's condition and his environment" $\left(\mathrm{ED}_{3}\right)$. "To assess the patient's respiration, it is necessary to have video data" $\left(E D_{1}\right)$, "but even a video cannot provide an impression of fine shades of the skin due to the influence of artificial light" $\left(E_{4}\right)$ or "replace the tactile impression a doctor senses by touching the patient's skin” $\left(E D_{8}\right)$. All interviewed emergency doctors basically concede the need and the potential of the teledoctor concept, however, according to their statements, the success of the teledoctor relies on reliable technical equipment.

In our sample, there were also participants who have already had some telemedical experience. They remarked that the usage of a teledoctor is shifting the main focus in an emergency operation: the patient will not be exclusively in the center of attention, and the operation will become less personal $\left(\mathrm{ED}_{8}\right)$, but the patient is receiving a qualitatively better treatment.

\footnotetext{
"It [the teledoctor concept] will be a support for the patient because a faster and more targeted information flow [...] means a better scheduling and preparation for the hospital personnel. Not so much time will be wasted during the information transfer from ambulance to hospital and in the further processing" $\left(\mathrm{ED}_{9}\right)$.
}

Regarding the scenario, in which an emergency doctor on-site consults a teledoctor (Scenario 1), the patient benefits not only from the expedited information flow, but also from two doctors taking care of him. The expertise of two medical professionals and the access to advanced information takes EMS on a qualitatively higher level. This emergency doctor-teledoctor-relation contains many benefits, but also reveals a competence problem: Both doctors are having the same qualification and are at the same time in charge. As an emergency differs from case to case, the doctor on-site may vary the standardized schemes what could contradict the advise of the teledoctor. Also, another barrier is that "the emergency doctor on-site feels observed, controlled, disturbed, and also patronized $\left(\mathrm{ED}_{3}\right)$ )"

Moreover, integrating an actor in a group interaction who is not physically on-site leads to miscellaneous communication problems.

\footnotetext{
"The voice connection between teledoctor and emergency doctor on-site confuses other people at the incident site. They do not know to whom he [emergency doctor on-site] is talking and why he is talking at all“ $\left(\mathrm{ED}_{8}\right)$.
}

The rescue team sometimes does not know to whom the doctor talks or if he is receiving a message on his headset $\left(\mathrm{ED}_{8}, \mathrm{ED}_{10}\right)$. Communication paths, and turn-taking between teledoctor and ED on-site needs extra-training:

„You have to learn to let people finish speaking, and to announce [communicative actions] before asking a question as each question may interrupt actions on-site and deflect [actors'] attention. [...] You need to develop an own communication culture“ $\left(\mathrm{ED}_{10}\right)$. 
But, the verbal communication via headsets causes confusion also for the patient. For instance, elderly people who are rarely used to ICT feel irritated by this. In case of a psychiatric emergency, it could even impair the situation $\left(\mathrm{ED}_{3}, \mathrm{ED}_{9}\right)$.

\subsection{Quantitative Findings (Questionnaire)}

As it was an exploratory study with only a small sample of participants, statistical analyses seemed not to be appropriate. Instead, we descriptively analyzed the data.

6.2.1 Which medical operations could be supported by ICT during a rescue operation?

Emergency doctors' answers in this section were quite clear-cut: ICT would be very helpful for an onsite diagnosis by means of physiological data (mainly heart measures). $40 \%$ of participants rate ICT support as very high, and $20 \%$ as high. Another main benefit of ICT was seen in the access to online data and medical libraries (confirmed by more than $70 \%$ of participants). A next service, for which ICT was seen as extremely helpful, is the (online) contact to the hospital (30\% report the utility of ICT as high, $50 \%$ as very high). With respect to the support of documentation, the sample was quite undecided. Finally, ICT was judged to be extremely helpful to have the possibility to reach an experienced colleague at any time (very high: 18\%, high: 40\%).

ICT usage was rated as less helpful regarding the contact to the general practitioner or the accessibility to therapy guidelines during the emergency operation,.

\subsubsection{How big are expected gains by the usage of} telemedical services in emergency medicine?

Emergency doctors also had to rate the expected gains of ICT usage within the emergency rescue operation. Figure 3 shows the descriptive results.

As can be seen from Figure 3, there is a basic positive evaluation of perceived benefits, taken from the fact that the benefit in all items were rated with at least medium ( $\mathrm{M}=3$ points). Perceived benefits cover a broad range of arguments, ranging from medical diagnosis quality, documentation accuracy, and a higher efficiency (time and costs) up to a decrease of false alarms due to a more time-critical information and communication procedure.

However, it is also noticeable, that none of the gains are expected to be very high ( $\mathrm{M}=6$ points) or even high ( $M=5$ points). Apparently, experts are quite reluctant in fully believing ICT being very beneficial.

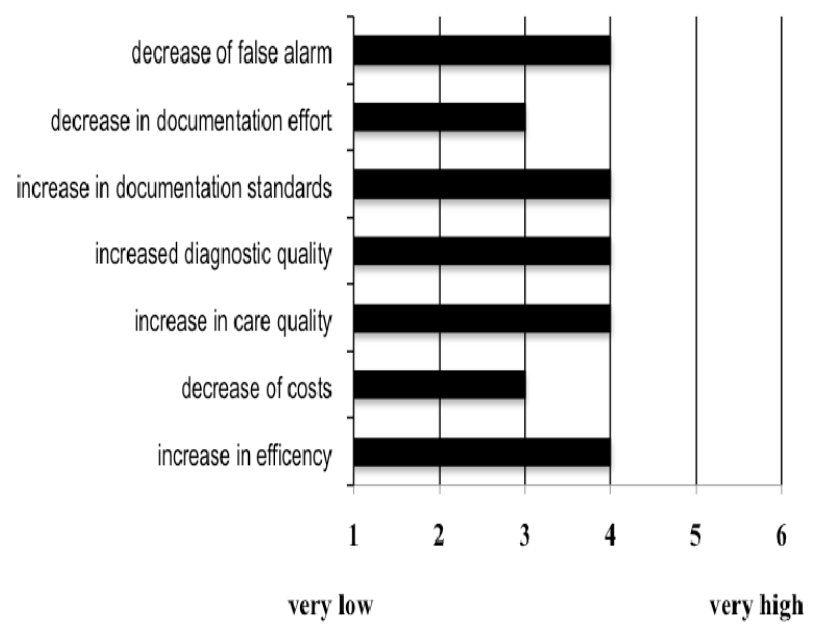

Figure 3. Perceived benefits (mean ratings) of ICT usage within the emergency rescue chain.

6.2.3 How big are expected drawbacks by the usage of telemedical services in emergency medicine

In order to understand their reluctance, the perceived drawbacks should be referred to. This is undertaken in the next analysis. Descriptive outcomes can be taken from Figure 4.

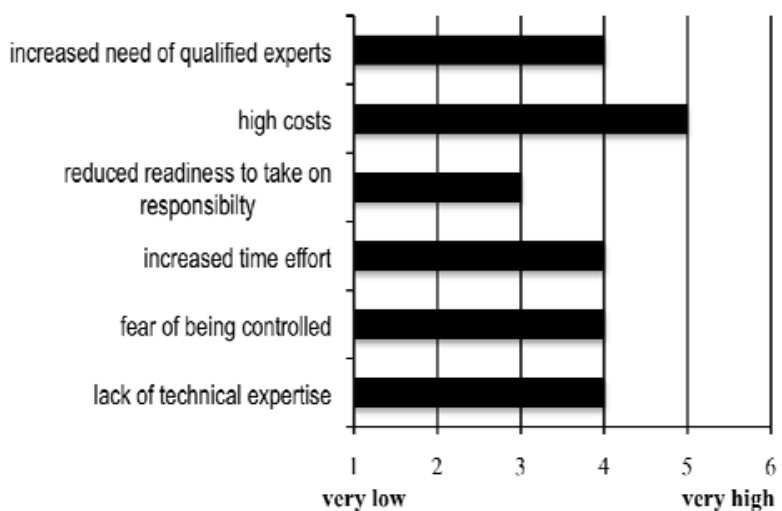

Figure 4. Perceived drawbacks (mean ratings) of ICT usage within the emergency rescue chain

Figure 4 illustrates that there is a number of potential pitfalls when using telemedical services in the emergency rescue operation. The strongest drawback is seen in the increase of costs, which are assumed to come along with the ICT implementation. Participants also suppose that the usage of ICT is time consuming and necessarily have. In fact, the vocational training for emergency doctors does not focus on the handling competence of ICT, even though ICT is a necessary requirement in an emergency doctors' daily routine. Participants also confirmed the fear of being controlled through ICT, which is noteworthy. It shows that ICT - as beneficial as it might be out of medical or emergency reasons - has drawbacks as the emergency doctor 
with his/her high responsibility and the enormous time pressure on-site, fears to be in charge of potential "errors" or unfortunate circumstances. This is a serious problem, as technology usage in high emergency situations requires a sensitive and novel conceptualization of legal and ethical issues.

\subsubsection{Does the potential benefit by telemedical services outperform the potential costs?}

So far we reported on potential drawbacks and benefits. It was found that emergency doctors have positive and negative expectations at the same time. In order to understand the "net" acceptance of telemedical services, we asked whether the perceived benefits outperform the expected drawbacks. This is

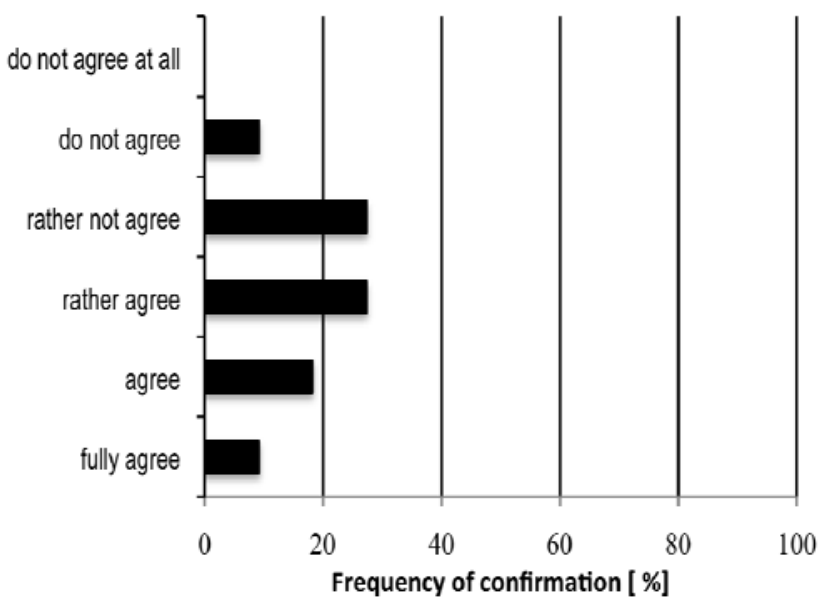

pictured in Figure 5.

Figure 5. Percentage of participants confirming the question: Does the potential benefits of ICT outperform the expected drawbacks?

This question was not answered clear-cut, even though - overall - there was a tendency to confirm the question (more participants confirmed than denied it). Obviously, emergency doctors' expected drawbacks have to be taken seriously and should be carefully considered in future efforts to implement ICT in the emergency workflow.

\section{Discussion and Conclusion}

The evaluation of the teledoctor as a possible telemedical support for emergency situations basically showed a positive result. However, there were also shortcomings and concerns from the perspective of emergency doctors. Basically, any technical support in the high-demanding and timecritical rescue-chain is a relief to the emergency staff involved in the process. There is no doubt, and all interviewed emergency doctor agree on this, the teledoctor is a powerful concept with a high usefulness. The teledoctor is able to compensate a lack of emergency physicians on-site, to support less experienced colleagues (with a possibly) different specialization, to context-adaptively advise paramedics as well as to authorize medical treatment. Also, respondents agreed on the increasing efficiency of the emergency process: by the help of a teledoctor, the diagnosis on-site can be accomplished by means of a more detailed and targeted medical information, which basically expedites the logistics and the organization of the rescue operation.

However, the interviewed emergency doctors also reported shortcomings and barriers towards the usage of a teledoctor. One concern is the low trust in the reliability of the technology involved. The second one is that a face-to-face experience with an emergency patient on-site seems to be a unique feature, which is - from the perspective of an "old stager” emergency physician - not replaceable by any digital medium. Neither a photo of a patient (too static) nor a video is sufficient to "feel" the actual condition of an emergency patient. Another concern was the alleged attention shift from the emergency situation and the patient as such to the advice or discussion with the teledoctor. Finally, also conflicts and struggling for competence had been expected by the interviewed doctors whenever the advise of the teledoctor contradicts the diagnosis and the impression of the emergency personnel on-site. With respect to optimization of the emergency situation, the teledoctor concept seems to be a promising way of meeting the upcoming challenges in emergency medicine.

Future studies will have to examine if the concerns raised by the emergency doctors will vanish with increasing experience in both roles, as emergency doctor on-site as well as a supervising teledoctor in the primary control unit remotely.

In addition, there are two major points, which seem to be crucial for future effort to increase electronic telemedical services in the EMS workflow.

One is that emergency doctors report not to automatically have the full competence when handling technology. From our all daily experience we know that a low usability and a high cognitive complexity of ICT interfaces are painful and do considerably hinder an efficient and satisfying interaction with technology. For emergency doctors, who are not especially educated in the use of technology, a low usability and a higher effort to use the devices in a time critical and life-threatening situation, this is even more critical.

The second point goes hand in hand with the first and represents a serious concern of emergency doctors: the fear that they could be controlled by means of ICT. Despite being obviously advantageous for the diagnosis efficiency and quality, ICT may also be a problem for emergency doctors. It allows a continuous recording of all actions on site and could 
be used for a post-hoc recrimination and blaming of doctors, whenever it came to unfortunate rescue operations.

While it is naturally justifiably to blame clear medical mistakes, a cautionary note regards the implications of ICT usage in the emergency rescue, for which legal framework conditions are not yet valid. Technology usage in novel contexts makes it necessary to adapt or even renew or create legal regulations to clarify responsibilities and protect patients, their relatives, but at the same time also emergency doctors.

\section{Acknowledgements}

We owe gratitude to the emergency doctors, who volunteered to take part in this study. Thanks also to our research partners from the University Hospital Aachen, Prof. Dr. Rolf Rossaint, Dr. Max Skorning, and Daniel Wielpütz, for their profound knowledge of the emergency medicine field as well as efficient organizational support. Also, we thank Luisa Bremen, Simon Himmel, Oliver Sack, and Stefan Ladwig for their research support. This work was funded by the Excellence initiative of German State and Federal governments.

\section{References}

[1] Behrendt, H., \& Runggaldier, K. (2009). Ein Problemaufriss über den demographischen Wandel in der Bundesrepublik Deutschland. Notfall \& Rettungsmedizin , 12 (1), 45-50.

[2] Prückner, S., \& Madler, C. (2009). Der demographische Wandel. Notfall \& Rettungsmedizin 12 (1), 13-18.

[3] Na, I.-S., Skorning, M. May, A., Schneiders, M.-T., Protogerakis, M., Beckers, S., Fischermann, H., Brodziak, T. \& Rossaint, R. (2011). Med-on-@ix: Real-time Teleconsultation in Emergency Medical Services Promising or Unnecessary? In M. Ziefle \& C. Röcker, (eds.). Human-Centered Design of eHealth Technologies. Hershey, P.A., IGI Global.

[4] Arcury TA, Preisser JS, Gesler WM, Sherman JE (2004). Complementary and Alternative Medicine Use among Rural Residents in Western North Carolina. Complementary Health Practice Review. 9, 93-102.

[5] Bronstein JM, Morissey MA. (2008). Determinants of Rural Travel Distance for Obstetrics Care. Medical Care. 28, 853-855.

[6] Jemmet, M.E, Kendal K.M., Fourre, M.W., Burton J.H. (2003). Unrecognized misplacements of endotracheal tubes in a mixed urban to rural emergency medical service setting. Academics Emergency Medicine, 10, 961-965

[7] Gries, A., Helm, M.\& Martin, E. (2003). Zukunft der präklinischen Notfallmedizin in Deutschland. Anästhesist, 52: 718-724.

[8] Chu, Y., Ganz, A. (2004). A mobile teletrauma system using 3G networks. IEEE Trans Inf Technol Biomedicine, 8(4), 456-462.

[9] Erder, M.H., Davidson, S.J., Cheney, R.A. (1989) Online medical command in theory and praxis. Annals of Emergency Medicine, 18, 261-268.
[10] Wuerz, R.C., Swope, G.E., Holliman C.J., Vazquezde Miguel, G. (1995) Online medical direction: a prospective study. Prehospital Disaster Medicine, 10, 174177.

[11] Adams, G.L., Campbell, P.T., Adams, J.M. et al (2006). Effectiveness of prehospital wireless transmission to a cardiologist via handheld device for patients with acute myocardial infarction. American Journal of Cardiology, 98, 1160-1164.

[12] van Halteren, A., Bults, R., Wac, K., Dokovsky, N., Koprinkov, G., Widya, I., Konstantas, D., Jones, V., Herzog, R. (2004). Wireless body area networks for healthcare: the MobiHealth project. Studies of Health Technolology Informaticsm 108, 181-193.

[13] Walderhaug, S., Meland, P., Mikalsen, M., Sagern, T. \& Brevik, J. (2008). Evacuation support system for improved medical documentation and information flow in the field. International Journal of Medical Informatics, 77 (2), 137-151.

[14] McCurdy, N.J., Griswold, W.G., \& Lenert, L.A. (2005). RealityFlythrough: enhancing situational awareness for medical response to disasters using ubiquitois video. Proceedings of the American Medical Informatics Association, 510-514.

[15] Kyng, M., Nielsen, E., \& Kristensen, M. (2006). Challenges in designing interactive systems for emergency response. Proceedings of the 6th conference on Designing Interactive systems, 301-310.

[16] Li, J., Wilson, L., Stapleton, S., \& Cregan, P. (2006). Design of an advanced telemedicine system for emergency care. Proceedings of the 18th Australia conference on Computer-Human Interaction: Design: Activities, Artefacts and Environments, 416.

[17] Reddy, M., Paul, S., Abraham, J., McNeese, M., DeFlitch, C., \& Yen, J. (2009). Challenges to effective crisis management: Using information and communication technologies to coordinate emergency medical services and emergency department teams. International Journal of Medical Informatics, 78 (4), 259-269.

[18] Berg, M. (1999) Patient care information systems and healthcare work: A socio-technical approach. International Journal of Medical Informatics, 52(2), 87-101.

[19] Aarts, J., Peel, V. \& Wright, G. (1998). Organizational Issues in health informatics: a model approach. International Journal of Medical Informatics, 52(1-3), 235-242.

[20] Manoj, B.S. \& Hubenko, A. (2007). Communication challenges in emergency response. Communication of the ACM, 50(3), 51-53.

[21] Wears, R., \& Perry, S. (2002). Human factors and ergonomics in the emergency department. Annals of Emergency Medicine, 40 (2), 206-212.

[22] Arning, K. \& Ziefle, M. (2009): Cognitive and personal predictors for PDA navigation performance. Behaviour and Information Technology, 28(3), 251-263.

[23] Ziefle, M. (2002). The influence of user expertise and phone complexity on performance, ease of use and learnability of different mobile phones. Behaviour and Information Technology, 21(5), 303-311.

[24] Ziefle, M. \& Schaar, A.K. (2010). Technical Expertise and its Influence on the Acceptance of Future Medical Technologies. What is influencing what to which extent? In G. Leitner, et al. (eds). HCI in Work \& Learning, Life \& Leisure, Human-Computer Interaction and Usability Engineering of the Austrian Computer Society, USAB 2010, LNCS 6389 (pp. 82-100). Berlin, Heidelberg: Springer. 
[25] Arning, K. \& Ziefle, M. (2009). Different perspectives on technology acceptance: The role of technology type and age. In A. Holzinger \& $\mathrm{K}$. Miesenberger (Eds.). Human-Computer Interaction for eInclusion. USAB 2009 (pp. 20-41), LNCS 5889; Berlin: Springer.

[26] Beul, S., Mennicken, S.; Ziefle, M. Jakobs, E.-M.; , Wielpütz, D.; Skorning, M. \& Rossaint, R. (2010). The impact of usability in emergency telemedical services. In V. Duffy (ed.). Advances in Human Factors and Ergonomics in Healthcare. Boca Raton: CRC Press.

[27] Wilkowska, W. \& Ziefle, M. (2009). Which factors form older adults' acceptance of mobile information and communication technologies? In A. Holzinger and $\mathrm{K}$. Miesenberger (Eds.). Human-Computer Interaction for eInclusion. USAB 2009 (pp. 81-101), LNCS 5889; Berlin: Springer.

[28] Wilkowska, W. \& Ziefle, M, (2011). User diversity as a challenge for the integration of medical technology into future home environments. In M. Ziefle \& C. Röcker (eds). Human-Centred Design of eHealth Technologies. Concepts, Methods and Applications (pp. 95-126). Hershey, P.A. IGI Global.

[29] Röcker, C. \& Ziefle, M. (2011): E-Health, Assistive Technologies and Applications for Assisted Living: Challenges and Solutions. Hershey, P.A.; IGI Global.

[30] Ziefle, M. \& Röcker, C. (2011): Human-Centered Design of E-Health Technologies: Concepts, Methods and Applications. Hershey, P.A.; IGI Global.

[31] Trout, A., Magnusson, A., Hedges, J. (2000). Patient Satisfaction Investigations and the Emergency Department: What Does the Literature Say? Academic Emergency Medicine, 7 (6), 695-709.

[32] Chisholm, C.D., Collisson, E.K.; Nelson, D.R. \& Cordell, W.H. (2000). Emergency Department Interruptions: Are Emergency Physicians "Interruptdriven" and "Multi-tasking"? Academic Emergency Medicine, 7(11), 1239-1243. 